\title{
CARACTERIZAÇÃO FÍSICA E SENSORIAL DAS BOLACHAS REDONDAS PRODUZIDAS E COMERCIALIZADAS NO MUNICÍPIO DE CURRAIS NOVOS/RN
}

\author{
Maria Luísa Rocha de Macedo \\ Pesquisas Aplicadas em Ciência e Tecnologia de Alimentos, IFRN, Campus Currais Novos, \\ Bolsista Programa de IC, aluna regular do Curso Técnico em Alimentos modalidade \\ Integrado. E-mail: maria-luisa-rocha@ hotmail.com \\ Odisséia Carla Pires Gaspareto \\ Pesquisas Aplicadas em Ciência e Tecnologia de Alimentos, IFRN, Campus Currais Novos, \\ Professora do curso Técnico em Alimentos. \\ odisseia.gaspareto@ifrn.edu.br \\ Dayene Louyse Lírio Dantas \\ Pesquisas Aplicadas em Ciência e Tecnologia de Alimentos, Técnica em alimentos. \\ louyse_lirio@hotmail.com
}

\section{RESUMO}

Os biscoitos populares são produtos amplamente consumidos nas cidades do interior do estado e até em sua capital. No município de Currais Novos, a maioria das padarias e mercados possui fabricação própria, o que diversifica a distribuição e aumenta a concorrência entre as marcas. Dentre os biscoitos mais conhecidos e apreciados estão as bolachas redondas. O presente trabalho tem como objetivo caracterizar esse tipo de alimento quanto aos parâmetros físicos, envolvendo determinações de massa, comprimento, espessura, largura, volume, volume específico, densidade e expansão; e sensoriais, tais como cor, sabor, textura, aroma e aparência, assim como o teste de aceitação. Com os resultados obtidos, verificou-se que os parâmetros físicos que estão mais uniformes entre as marcas são densidade $(0,27 \mathrm{~g} / \mathrm{mL})$, expansão $(0,23 \mathrm{~cm} / \mathrm{mm})$ e comprimento $(3,41 \mathrm{~cm})$, com desvios padrão de, respectivamente, $0,02,0,02$ e 0,22 . Os que apresentam as maiores disparidades são espessura $(14.89 \mathrm{~mm})$, largura $(24,9 \mathrm{~mm})$ e volume $(7,56 \mathrm{~mL})$, com $1,05,1,21$ e 1,32 de desvio padrão. Quanto às análises sensoriais, a Marca 4 foi rejeitada em todos os quesitos e a Marca 5 obteve o maior grau de aceitação, sendo aprovada em todos os fatores observados.

PALAVRAS-CHAVE: biscoitos populares, bolachas redondas, análise física, análise sensorial

\section{PHYSICAL AND SENSORY ANALYSIS OF ROUND CRACKERS PRODUCED AND MARKETED IN THE TOWN OF CURRAIS NOVOS/RN}

\begin{abstract}
Popular cookies are products largely consumed in the countryside cities of the state and even in its capital. In the town of Currais Novos most bakeries and stores have their own production, what diversifies the distribution and increases the brands competition. Among the most popular and appreciated cookies are the round ones. This work has as its objective to evaluate this kind of food, its physical standards, involving body-mass determination, length, thickness, width, volume, specific volume, density and expansion; and sensorial like color, taste, texture, scent and appearance, as its acceptance test. With the obtained results, it was verified that the physical standards which are mostly uniforms between the brands are density
\end{abstract}


$(0,27 \mathrm{~g} / \mathrm{mL})$, expansion $(0,23 \mathrm{~cm} / \mathrm{mm})$ and length $(3,41 \mathrm{~cm})$, with patterns detours of, respectively, $0,02,0,02$ e 0,22 . Those who show the huges differences are thickness $(14.98$ $\mathrm{mm})$, width $(24,9 \mathrm{~mm})$ and volume $(7,56 \mathrm{~mL})$ with $1,05,1,21$ e 1,32 of pattern detour. About the sensorial analysis, the brand number 4 was rejected in every item, and brand number 5 had the highest rate of acceptance, being approved in every observed factor.

KEY-WORDS: popular cookies, round crackers, physical analysis, sensorial analysis. 


\section{CARACTERIZAÇÃO FÍSICA E SENSORIAL DAS BOLACHAS REDONDAS PRODUZIDAS E COMERCIALIZADAS NO MUNICÍPIO DE CURRAIS NOVOS/RN}

\section{INTRODUÇÃO}

O mercado de biscoitos do Brasil passou por grandes transformações na década de 1990, fazendo com que o país abrisse suas fronteiras para a importação da farinha, matéria-prima essencial na fabricação dos biscoitos. Esse fato determinou o aumento da concorrência, uma vez que a importação de biscoito foi incentivada pelo governo.

Com a concorrência, as indústrias brasileiras investiram em qualidade, melhorando seus processos e produtos. Este fato fez com que os produtos importados tivessem suas vendas diminuídas.

O consumo de biscoitos aumentou, mas $45 \%$ destas vendas provêm de pequenas e médias empresas. Segundo dados do SEBRAE (2008), o Brasil apresentou, em 2007, 1271 pequenos e micro fabricantes de biscoitos, concentrados, sobretudo em Minas Gerais (21\%), São Paulo (16,5\%), Rio Grande do Sul (11\%), Bahia (9\%) e Santa Catarina (8\%), que, juntos, totalizam 2/3 dos fabricantes de biscoitos do Brasil. O Rio Grande do Norte detém 2,7\% destas fábricas.

Apesar do mostrado anteriormente, o Nordeste é a região que mais consome biscoitos $(36,1 \%)$. Esse alto índice de consumo é confirmado pelos dados da Pesquisa de Orçamentos Familiares (POF), realizada pelo IBGE em 2003.

Segundo SEBRAE-ES, os biscoitos populares são destinados a pessoas de poder aquisitivo mais baixo, sendo encontrados preferencialmente nas periferias das cidades e nas regiões rurais.

O mercado de biscoitos populares possui um grande número de concorrentes que fabricam produtos idênticos ou muito similares.

Com o intuito de identificar os perfis físicos e sensoriais destes produtos, o presente trabalho estudou as bolachas redondas vendidas em mercados e padarias do município de Currais Novos/RN.

\section{MATERIAIS E MÉTODOS}

Para a escolha da matéria-prima foi utilizado um questionário com 7 (sete) perguntas que tinham como objetivo avaliar a preferência dos consumidores em relação aos biscoitos populares. Foram entrevistadas 110 pessoas em 6 pontos comerciais na cidade de Currais Novos, distribuídos em 3 bairros.

As marcas de bolachas redondas escolhidas foram adquiridas no comércio local e tiveram os seguintes parâmetros analisados: massa (pesada diretamente em balança eletrônica digital semi-analítica - 200g); comprimento, espessura e largura (medidos com paquímetro); volume (segundo técnica de SILVA; SILVA; CHANG (1998) e FERREIRA (2002) apud FERREIRA E AMICI (2009)); volume específico (mL/g); densidade $(\mathrm{g} / \mathrm{mL})$; e expansão $(\mathrm{cm} / \mathrm{mm})$.

Para as análises sensoriais utilizou-se teste de aceitação realizado com 40 provadores, de ambos os sexos, selecionados de forma aleatória. Os provadores utilizaram para a avaliação a escala hedônica estruturada com valores de 1 (desgostei muitíssimo) a 9 (gostei muitíssimo) 
onde foram avaliados os seguintes quesitos: cor, sabor, textura, aroma e aparência, assim como o teste de aceitação. O teste de Tukey foi utilizado para se determinar a diferença significativa entre as amostras em nível de significância de 5\%.

\section{RESULTADOS E DISCUSSÕES}

\section{1) Escolha do Biscoito}

Quando os entrevistados foram perguntados sobre a frequência de consumo dos biscoitos populares (Figura 1), 78\% alegaram consumir as bolachas redondas todos os dias. Os consumidores também foram questionados sobre a "marca preferida" e estes puderam citar as marcas que mais consomem. Desta forma, observou-se (Figura 2) que a marca intitulada como " 2 " foi a que obteve a maior preferência do consumidor com $34 \%$ das respostas. Para preservar o nome das marcas, foram atribuídos pseudônimos numerais.

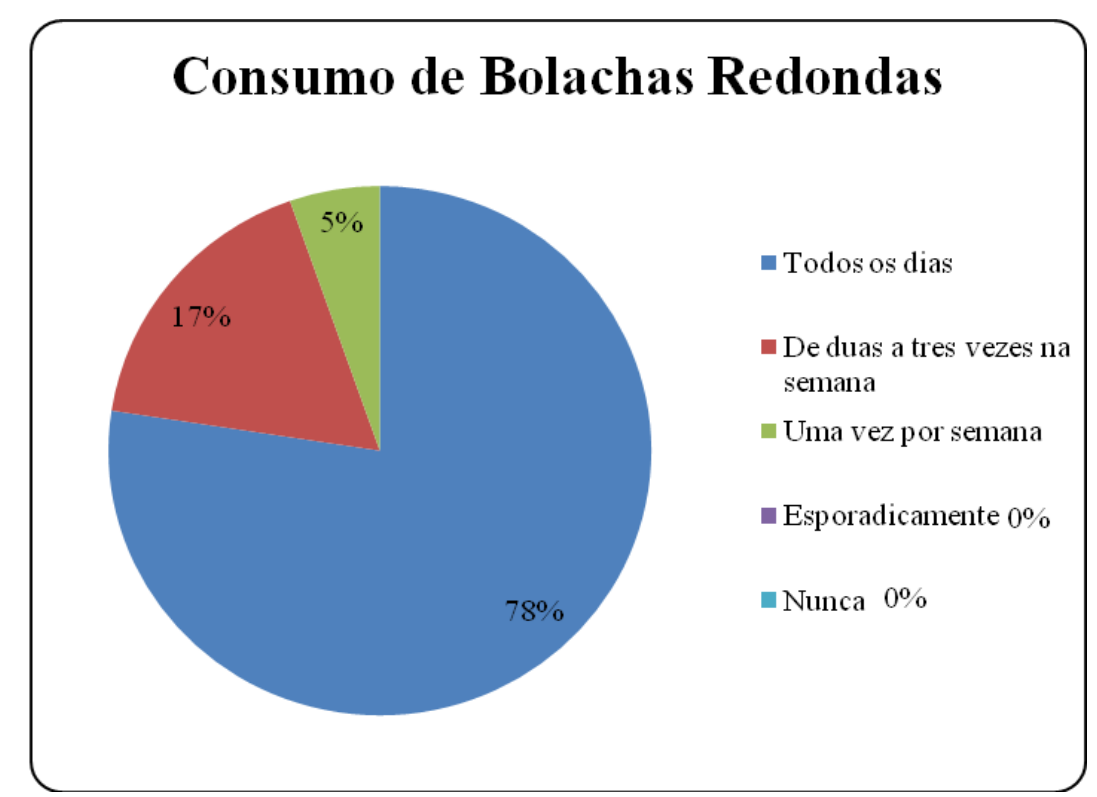

Figura 1 - Gráfico de Preferência das marcas de bolachas redondas.

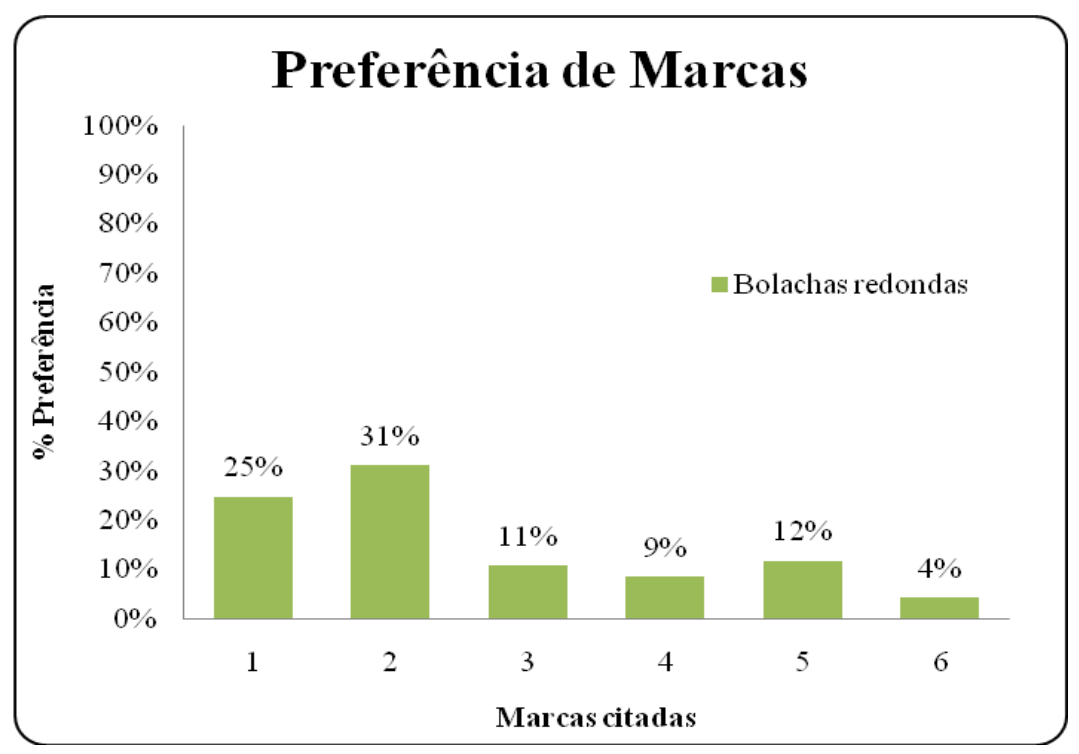

Figura 2 - Gráfico de Preferência das marcas de bolachas redondas. 


\section{2) Análises físicas}

Para as análises físicas, foram utilizadas amostras representativas de $10 \%$ do total de bolachas contidas em 2 (dois) pacotes de cada marca, escolhidas aleatoriamente, e verificados os parâmetros físicos, conforme descritos em Metodologia. A partir dos resultados obtidos, foram calculadas as médias de cada marca e, posteriormente, as médias entre todas as marcas e seus respectivos desvios-padrão, como pode ser visto na Tabela 1.

Tabela 1 - Médias das análises físicas das bolachas redondas

\begin{tabular}{|l|r|r|r|r|r|r|r|}
\hline Análises / Médias & $\begin{array}{c}\text { Marca } \\
\mathbf{1}\end{array}$ & $\begin{array}{c}\text { Marca } \\
\mathbf{2}\end{array}$ & $\begin{array}{c}\text { Marca } \\
\mathbf{3}\end{array}$ & \multicolumn{1}{c|}{$\begin{array}{c}\text { Marca } \\
\mathbf{4}\end{array}$} & \multicolumn{1}{c|}{$\begin{array}{c}\text { Marca } \\
\mathbf{5}\end{array}$} & $\begin{array}{c}\text { Marca } \\
\mathbf{6}\end{array}$ & $\begin{array}{c}\text { Médias } \pm \\
\text { Desvio Padrão }\end{array}$ \\
\hline Massa (g) & 2,03 & 1,52 & 2,04 & 2,92 & 1,64 & 2,57 & $2,11 \pm 0,54$ \\
\hline $\begin{array}{l}\text { Comprimento } \\
(\mathrm{cm})\end{array}$ & 3,33 & 3,37 & 3,23 & 3,33 & 3,27 & 3,82 & $3,41 \pm 0,22$ \\
\hline Espessura (mm) & 14,39 & 16,09 & 14,3 & 14,39 & 14,51 & 15,3 & $14,89 \pm 1,05$ \\
\hline Largura (mm) & 26,62 & 23,86 & 23,31 & 25,17 & 24,08 & 25,97 & $24,9 \pm 1,21$ \\
\hline Volume (mL) & 7,03 & 6,22 & 7,16 & 9,47 & 6,44 & 8,85 & $7,56 \pm 1,32$ \\
\hline $\begin{array}{l}\text { Volume específico } \\
\text { (mL/g) }\end{array}$ & 3,49 & 4,02 & 3,61 & 3,27 & 3,98 & 3,48 & $3,64 \pm 0,33$ \\
\hline Densidade (g/mL) & 0,29 & 0,25 & 0,29 & 0,31 & 0,26 & 0,29 & $0,27 \pm 0,02$ \\
\hline $\begin{array}{l}\text { Expansão } \\
\text { (cm/mm) }\end{array}$ & 0,23 & 0,21 & 0,23 & 0,24 & 0,23 & 0,25 & $0,23 \pm 0,02$ \\
\hline
\end{tabular}

Com os resultados obtidos, tem-se que as maiores diferenças físicas entre as bolachas analisadas são encontradas na espessura, largura e volume, fato claramente evidenciado nos desvios-padrão da média geral das amostras e que também pode ser percebido calculando-se a amplitude das médias das marcas. Obteve-se ainda que a Marca 5 possui todos os valores próximos aos encontrados nas médias gerais, não apresentando nenhum extremo.

Quanto às relações entre os parâmetros analisados, percebe-se que a variação de volume influencia diretamente na massa e no volume específico, assim como a variação de densidade influencia na massa. Já a relação inversa está presente entre volume específico e massa, bem como densidade e volume.

\section{3) Análises sensoriais}

Observa-se na Figura 3, nos resultados das análises sensoriais, que a Marca 4 difere significativamente das demais marcas em todos os quesitos verificados na análise sensorial. Tem-se ainda que nos parâmetros textura e aroma não existe diferença significativa entre as marcas 1, 2, 3, 5 e 6. Quanto ao sabor, as marcas 4, 5 e 6 diferem entre si, bem como as amostras 4 e 5 no quesito cor. 


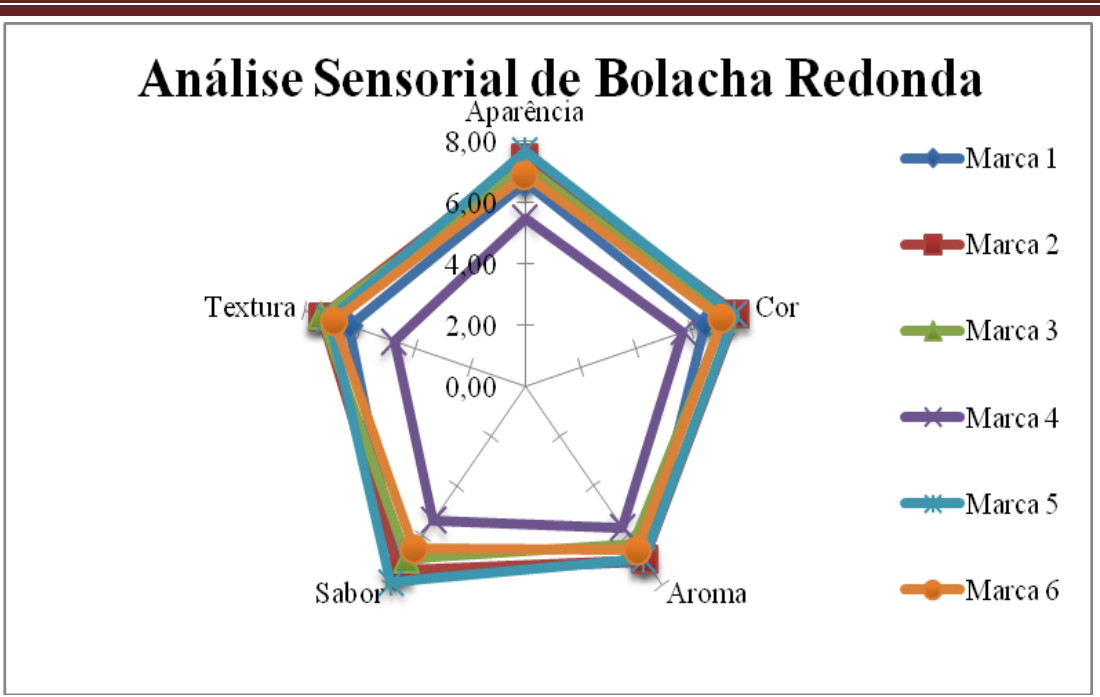

Figura 3 - Gráfico de análise sensorial de bolachas redondas

Quanto ao teste de aceitabilidade, que considera aceitas as amostras que obtiverem resultados superiores a 70\%, a Marca 4 foi reprovada em todos os quesitos observados, como pode ser visto na Figura 4. As únicas marcas que obtiveram valores maiores a $70 \%$ em todos os parâmetros verificados na análise sensorial foram a 2 e 5, sendo esta última a preferida dos provadores, com quase $90 \%$ de aprovação no quesito sabor.

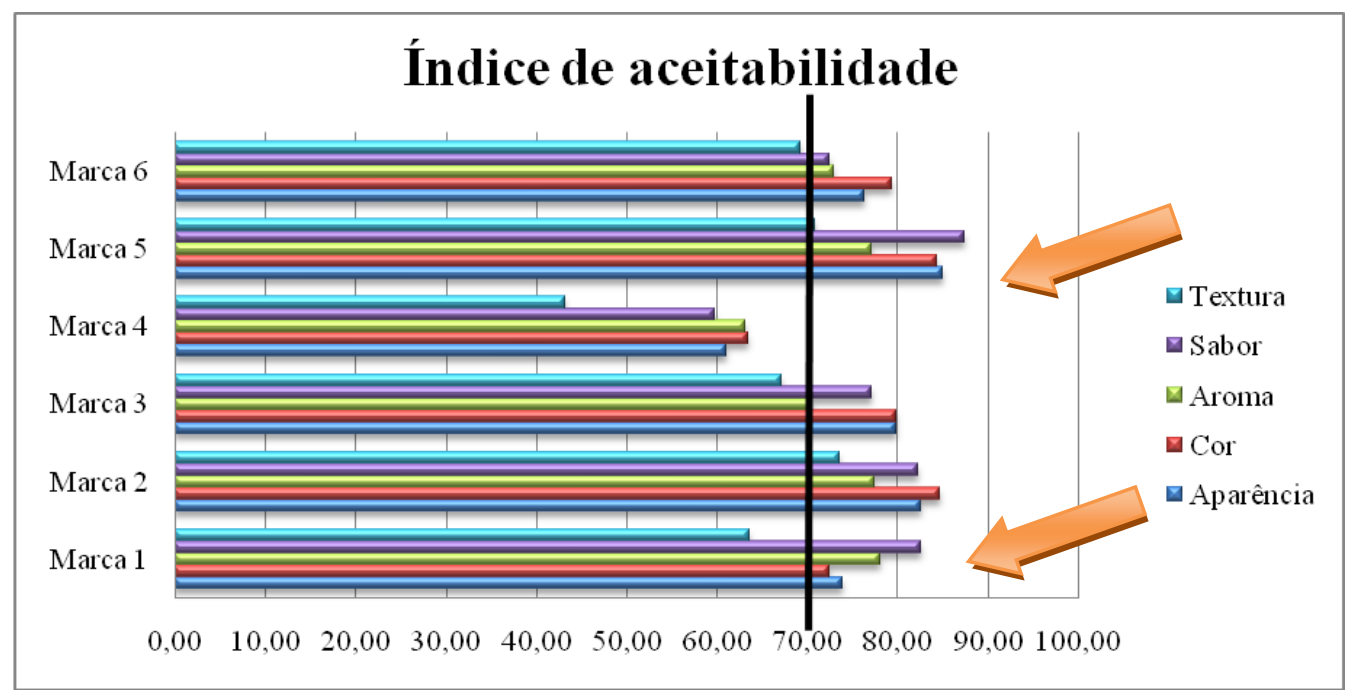

Figura 4 - Índice de aceitabilidade das bolachas redondas

\section{CONCLUSÕES}

As bolachas redondas são produtos consumidos diariamente por $78 \%$ dos munícipes de Currais Novos. Dos parâmetros medidos nas análises físicas, Volume $(\mathrm{mL})$ é o que apresenta mais variação entre as marcas e Comprimento $(\mathrm{cm})$ é o que apresenta maior uniformidade. $\mathrm{O}$ principal fator de escolha das bolachas foi a qualidade e a marca mais comprada foi a "1". Já na análise sensorial, a marca com maior aceitação foi a Marca 5, com 87,39\% de aceitabilidade (sabor). A marca 4 foi rejeitada em todos os quesitos observados na análise sensorial. 


\section{AGRADECIMENTOS}

Agradecemos ao IFRN por tornar esse projeto possível e aos que acreditam.

\section{REFERÊNCIAS}

1. FERREIRA, S. M. R.; AMICI, M. R. Caracterização física e sensorial de biscoitos. Higiene Alimentar, v.23 n.168/169, São Paulo, Jan./Fev., 2009.

2. PINHEIRO, P.R.; LIMA, D.P.; MELO, M.E.P. Otimização do Processo de Fabricação do Biscoito Tipo Cracker. Simpósio Brasileiro de Pesquisa Operacional. ISSN 1518-1731. Viçosa: Sociedade Brasileira de Pesquisa Operacional, 2000.

3. SEBRAE-ES; Fábrica de biscoito, Série perfil de projetos, Dez., 1999.

4. SEBRAE/ESPM; Biscoitos caseiros/não industrializados - Relatório Completo, Estudos de mercado, Set., 2008.

5. SILVA, M. R.; SILVA, M. A. A. P.; CHANG, Y. K. Utilização tecnológica dos frutos de jatobá-do-cerrado e de Jatobá-da-mata na elaboração de biscoitos fontes de fibra e isentos de açúcares. Ciência e Tecnologia de Alimentos, v.21 n.2, Campinas, Mai./Ago., 2001. 